Yu.L. Sayenko, D.N. Kalyuzhniy

\title{
IDENTIFICATION OF GENERALIZED LINEAR LOAD IN THREE-PHASE THREE-WIRE NETWORK IN THE PROBLEM OF THE DISTRIBUTION OF ACTUAL CONTRIBUTIONS AT THE POINT OF COMMON COUPLING
}

\begin{abstract}
Purpose. To perform structural and parametric identification of generalized load equivalent circuit of three-phase three-wire load in the network in the space of phase components. Methodology. Underlying structural identification methods are matrix analysis of electrical circuits. Parametric identification is based on the basic laws of electrical engineering. Results. The structure of a generalized load equivalent circuit is composed in three independent nodes. An approximate method for determining its parameters is proposed. The estimation error determination undistorted and distorted parts of the parameters of generalized load equivalent circuit. Originality. Approximate determination of equivalent circuit parameters are based on the results of a single measurement of voltages and phase currents. Practical value. The proposed replacement structure and a method for determining its parameters of the circuit can be used in the problem of the distribution of actual contributions at the point of common coupling. References 7, tables 1, figures 4.
\end{abstract}

Key words: equivalent circuit of linear generalized load, actual contribution, point of common coupling.

На основе матричного метода анализа электрических цепей определена структура эквивалентной схемы замещения обобщенной нагрузки. Предложен способ приближенного определения параметров эквивалентной схемы замещения обобщенной нагрузки. Выполнен анализ ошибок приближенного определения параметров эквивалентной схемы замещения обобщенной нагрузки применительно к задаче распределения фактических вкладов в точке общего присоединения. Библ. 7, табл. 1, рис. 4.

Ключевые слова: эквивалентная схема замещения линейной обобщенной нагрузки, фактический вклад, точка общего присоединения.

Introduction. The asymmetry and deviation of voltages is one of the main characteristics of electric power, which in most cases do not meet its quality [1]. Consequences of electrical consumption and lower quality of transmission power are well known [2], and, ultimately, to reduce financial losses [3]. Determination of the actual contributions to the reduction of electric power quality at the point of common coupling (PCC) is a solution of the problem of the distribution of compensation for economic loss to the injured party by all electricity market participants.

Problem definition. Existing methods for the distribution of actual contributions [4], irrespective of the implementation of the selected mathematical space, have a common problem. It is an adequate representation and parameterization of the generalized equivalent circuit load. So, in the space of generalized symmetrical components are load in the form of a set of current source or EMF with its resistance, which corresponds to the Norton or Tevenen equivalent circuits. In the space of phase components can restrict passive elements, where a clear opinion on the equivalent circuit of the structure in relation to the actual distribution of the contributions is not the problem. At asymmetrical load using the method of symmetrical components does not give any advantages over the phase space components. On this basis, the paper discusses the definition of the structure and parameters of equivalent circuit linear distorting the generalized threephase three-wire load in networks within the space of phase components.

The goal of investigations is to develop an approach for generalized linear identification in three-phase three-wire load in the network within the space of phase components for task allocation of actual contributions to the current of common coupling.
Results of investigations. We consider the power supply system (PSS) with a dedicated load relatively PCC which made measurement of indicators of quality of electric energy (Fig. 1,a). We assume that all the elements of the electric power system and electric energy receivers generalized stress can cause only linear (sinusoidal) voltage distortion. Then, the equivalent circuit can be represented PSS such as active and passive elements connection (Fig. 1,b). In addition, we take the assumption of neglecting the mutual impedance and conductivity of individual PSS components.

According to the method of nodal potentials the total equivalent circuit of power system and the generalized load described by the following matrix equation:

$$
\left\|\begin{array}{cc}
\boldsymbol{Y}_{a a} & \boldsymbol{Y}_{a b} \\
\boldsymbol{Y}_{b a} & \boldsymbol{Y}_{b b}
\end{array}\right\| \times\|\| \begin{gathered}
\boldsymbol{\Phi}_{a} \\
\boldsymbol{\Phi}_{b}
\end{gathered}\|=\| \begin{gathered}
\boldsymbol{J}_{a} \\
\boldsymbol{J}_{b}
\end{gathered} \|,
$$

where $a$ is the serial number of the last node from the side of the equivalent circuit of power system; $b$ is the number of independent nodes in the overall equivalent scheme of the electric power system and the generalized load.

The solution of equation (1) relative the sub-matrix $\overrightarrow{\boldsymbol{\Phi}}_{a}$ in the absence of active elements in the equivalent circuit of the generalized load is as follows:

$$
\left(\boldsymbol{Y}_{a a}+\boldsymbol{Y}_{e q}\right) \times \overrightarrow{\boldsymbol{\Phi}}_{a}=\overrightarrow{\boldsymbol{J}}_{a}
$$

where $\boldsymbol{Y}_{e q}$ is matrix of nodal conductivities of equivalent generalized load which characterizes the structure of its equivalent circuit.

The matrix $\boldsymbol{Y}_{e q}$ is determined as follows:

$$
\boldsymbol{Y}_{e q}=-\boldsymbol{Y}_{a b} \times \boldsymbol{Y}_{b b}^{-1} \times \boldsymbol{Y}_{b a} .
$$



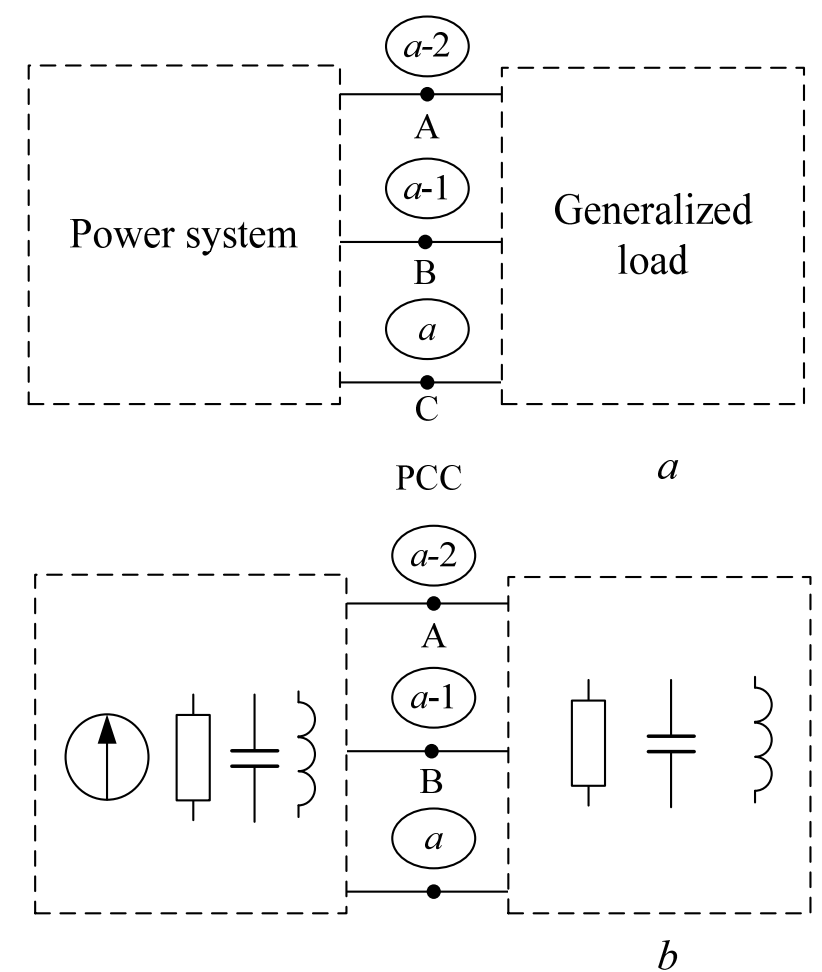

Fig. 1. Power supply system: $a$-structural circuit relative PCC; $b$ - schematic diagram of the replacement of the power system and the generalized load

A feature of the matrix $\boldsymbol{Y}_{e q}$ in our case is that all the elements in it, except for the block elements of the dimension [3×3], zero. This implies that an equivalent substitution of generalized load circuit must have three independent node. This condition corresponds to the structure of the equivalent circuit shown in Fig. 2.

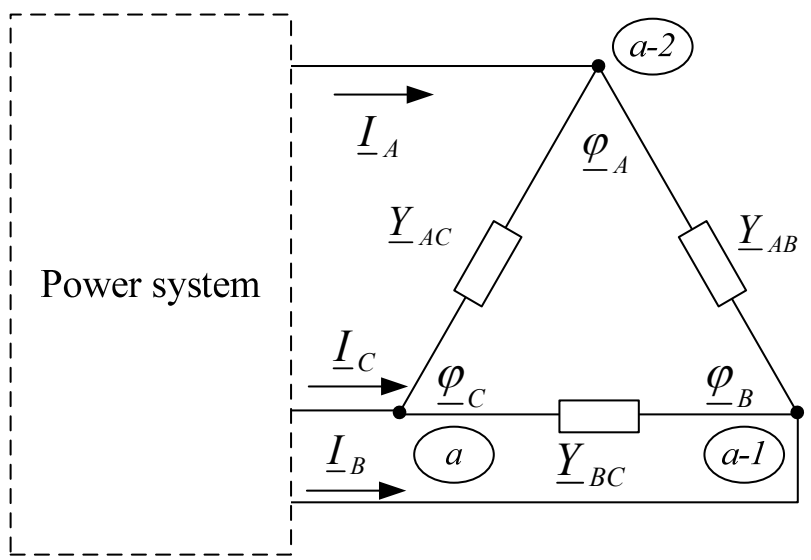

Fig. 2. Structure of the equivalent circuit of the generalized load

Let us consider a problem of the determination of the generalized load equivalent circuit parameters. Initial data are measured in the PCC phase currents $\left(\underline{I}_{A}, \underline{I}_{B}\right.$ and $\left.\underline{I}_{C}\right)$ and linear voltages $\left(\underline{U}_{A B}, \underline{U}_{B C}\right.$ and $\left.\underline{U}_{A C}\right)$.

Parameters of the equivalent circuit and measured mode parameters are interconnected as follows:

$$
\left\{\begin{array}{l}
\underline{U}_{A B} \cdot \underline{Y}_{A B}+0 \cdot \underline{Y}_{B C}+\underline{U}_{A C} \cdot \underline{Y}_{A C}=\underline{I}_{A} \\
\underline{U}_{B A} \cdot \underline{Y}_{A B}+\underline{U}_{B C} \cdot \underline{Y}_{B C}+0 \cdot \underline{Y}_{A C}=\underline{I}_{B} .
\end{array}\right.
$$

The system (4) consisting of two linear algebraic equations and containing three unknowns $\left(\underline{Y}_{A B}, \underline{Y}_{B C}\right.$ and $\left.\underline{Y}_{A C}\right)$ is uncertain and joint. Its general solution has the form

$$
\left\{\begin{array}{l}
\underline{Y}_{A B}=\left(\underline{I}_{A}-\underline{U}_{A C} \cdot \underline{Y}_{A C}\right) / \underline{U}_{A B} \\
\underline{Y}_{B C}=-\left(\underline{I}_{C}+\underline{U}_{A C} \cdot \underline{Y}_{A C}\right) / \underline{U}_{B C} .
\end{array}\right.
$$

To determine unknown conductivities $\underline{Y}_{A B}, \underline{Y}_{B C}$ and $\underline{Y}_{A C}$ it is possible to resort to a redefinition of the system (5) by the use of two independent data, for example, successive measurements. Applying the least squares method to such a system of equations, unknown conductivity will be determined as follows:

$$
\overrightarrow{\boldsymbol{Y}}=\left(\boldsymbol{U}^{T} \boldsymbol{U}\right)^{-1} \boldsymbol{U}^{T} \overrightarrow{\boldsymbol{I}}
$$

где $\boldsymbol{U}$ is the matrix of measured voltages; $\overrightarrow{\boldsymbol{I}}$ is the column matrix of measured currents; $\overrightarrow{\boldsymbol{Y}}$ is the column matrix of unknown conductivities.

Using the above approach determine the unknown conductivities requires compliance with the following conditions. Measured parameters mode must display two different PSS condition caused by changes only from the electric power system. From a practical point of view, this situation is unlikely in PSS so many researchers, for example, $[5,6]$, propose to conduct active experiment based on forced switching capacitor banks in the PCC. Taking into account the method of measuring the parameters of electrical energy quality, where the minimum measurement interval mode settings begins with $0.2 \mathrm{~s}$ this approach is unacceptable. In addition, the results of mathematical modeling show high sensitivity of the solution (6) to minor changes in the state of generalized load, as well as the magnitude of the disturbance from the power system. As a consequence, an adequate definition of the equivalent circuit parameters of the generalized load according to the expression (6) in a real operation is not technically feasible.

We consider the possibility of determining the approximate equivalent circuit parameters of the generalized load. For this purpose, we turn to its equivalent circuit represented as a star connection of elements (Fig. 3).

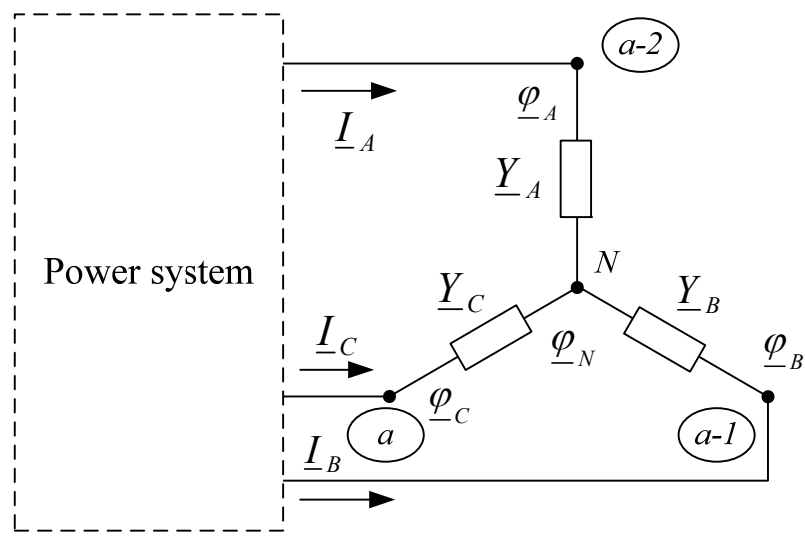

Fig. 3. Equivalent circuit of the generalized load with star connection of elements 
The considered equivalent circuit with four nodes is the described by the following system of three equations:

$$
\left\{\begin{array}{l}
\underline{U}_{A} \cdot \underline{Y}_{A}=\underline{I}_{A} \\
\underline{U}_{B} \cdot \underline{Y}_{B}=\underline{I}_{B} \\
\underline{U}_{C} \cdot \underline{Y}_{C}=\underline{I}_{C}
\end{array}\right.
$$

As it is known, an accurate measurement of the phase voltages of three-phase three-wire in the networks due to a physical lack of a neutral point, generally can not be performed. Therefore, for an approximate determination of the phase voltages use the measured values of the line voltages:

$$
\left\{\begin{array}{l}
\underline{U}_{A}^{\approx}=\left(\underline{U}_{A B 1} \cdot e^{-j 30}+\underline{U}_{A B 2} \cdot e^{j 30}\right) / \sqrt{3} ; \\
\underline{U}_{B}^{\approx}=\underline{U}_{B A}+\underline{U}_{A}^{\approx} ; \\
\underline{U}_{C}^{\approx}=\underline{U}_{C B}+\underline{U}_{B},
\end{array}\right.
$$

where $\underline{U}_{A B 1}$ and $\underline{U}_{A B 2}$ are the linear voltages of direct and inverse sequences, respectively.

The error of the determination of phase voltages for the equivalent circuit of Fig. 3 will be determined by the difference of exact $\underline{\varphi}_{N}$ and approximate $\left(U_{A}^{\approx}+\underline{U}_{B}^{\approx}+\underline{U}_{C}^{\approx}\right) / 3$ values of potentials of the point $N$ (Fig. 4):

$$
\Delta \underline{\varphi}=\underline{\varphi}_{N}-\left(\underline{U}_{A}^{\approx}+\underline{U}_{B}^{\approx}+\underline{U}_{C}^{\approx}\right) / 3
$$

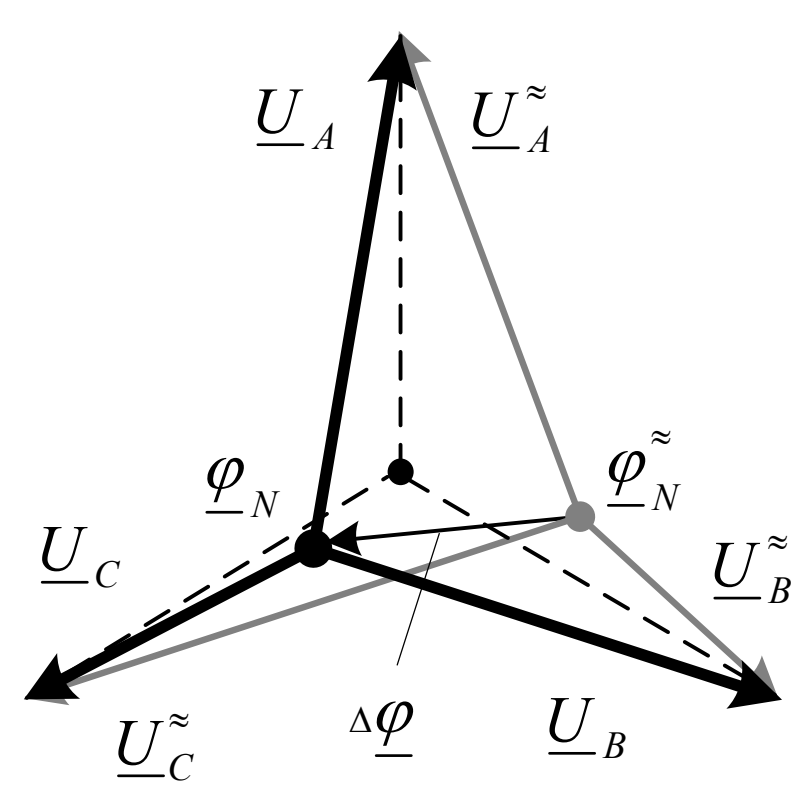

Fig. 4. Exact and approximate values of phase voltages for the equivalent circuit of the generalized load with star connection of elements (Fig. 3)

So, approximate values of conductivities $\underline{Y}_{A}^{\approx}, \underline{Y_{B}}$ and $\underline{Y}_{C}^{\approx}$, calculated by (7) taking into account (8) connected with their exact values by the following equality:

$$
\underline{Y}_{p h}^{\approx}=\underline{Y}_{p h}-\underline{Y}_{p h} \cdot \Delta \underline{\varphi} / \underline{U}_{p h}^{\approx},
$$

where the symbol " $p h$ " corresponds to phase A, B or C.

To solve the problem of the distribution of phase values the basic is dividing of the equivalent circuit into nondisturbing and disturbing parts. Here, from the point of view of phase conductivities determination, the most sensitive for this dividing are nondisturbing and disturbing parts used for the phase values determination by the voltage asymmetry:

$$
\left\{\begin{array}{l}
\underline{Y}_{p h}^{\text {nondis }}=\left(\underline{Y}_{A}+\underline{Y}_{B}+\underline{Y}_{C}\right) / 3 \\
\underline{Y}_{p h}^{\text {dis }}=\underline{Y}_{p h}-\underline{Y}_{p h}^{\text {nondis }}
\end{array}\right.
$$

From here, we conduct analysis of error of determination of nondisturbing and disturbing parts of the generalized load equivalent circuit parameters for two characterized cases when on buses of balanced and nonbalanced loads there is asymmetric voltage.

At the balanced load with asymmetric voltage errors of determination of $\underline{Y}_{p h}^{\text {nondis }}$ and $\underline{Y}_{p h}^{\text {dis }}$ are:

$$
\delta \underline{Y}_{p h}^{\text {nondis }}=\frac{\underline{Y} p h \cdot \Delta \underline{\varphi}}{3} \cdot\left(\frac{1}{\underline{U}_{A}^{\approx}}+\frac{1}{\underline{U}_{B}^{\approx}}+\frac{1}{\underline{U}_{C}^{\approx}}\right)
$$

$$
\delta \underline{Y}_{p h}^{d i s}=\underline{Y}_{p h} \cdot \Delta \underline{\varphi} / \underline{U}_{p h}^{\approx}-\frac{\underline{Y} p h \cdot \Delta \underline{\varphi}}{3} \cdot\left(\frac{1}{\underline{U}_{A}^{\approx}}+\frac{1}{\underline{U}_{B}^{\approx}}+\frac{1}{\underline{U}_{C}^{\approx}}\right) .
$$

We simplify the factor $\left(\frac{1}{\underline{U}_{A}^{\approx}}+\frac{1}{\underline{U}_{B}^{\approx}}+\frac{1}{\underline{U}_{C}^{\approx}}\right)$ assuming that $\left|\left(U_{A 2}^{\approx}\right) / \underline{U}_{A 1}^{\approx}\right|<<1:$

$$
\begin{gathered}
\left(\frac{1}{\underline{U}_{A 1}^{\approx}+\underline{U}_{A 2}}+\frac{1}{\underline{U}_{B}^{\approx}}+\frac{1}{\underline{U}_{C}}\right) \approx \\
\approx-\left(\frac{\underline{U}_{A 2}^{\approx}}{\left(\left(\underline{U}_{A 1}^{\approx}\right)^{2}\right.}+\frac{\underline{U} \tilde{B} 2}{\left(\underline{U}_{B 1}^{\approx}\right)^{2}}+\frac{\underline{U}_{C 2}^{\approx}}{\left(U_{C 1}\right)^{2}}\right)=-\frac{3 \cdot \underline{U}_{A 2}^{\approx}}{\left(\underline{U}_{A 1}\right)^{2}} .
\end{gathered}
$$

Then, taking into account (14) the approximate value of $\delta \underline{Y}_{p h}^{\text {nondis }}$ is:

$$
\delta \underline{Y}_{p h}^{\text {nondis }} \approx-\underline{Y}_{p h} \cdot \Delta \underline{\varphi} \cdot \frac{\underline{U}_{A 2}^{\approx}}{\left(\underline{U}_{A 1}^{\approx}\right)^{2}}
$$

Taking into account that factor $\underline{U}_{A 2}^{\approx} /\left(U_{A 1}^{\approx}\right)^{2}$ in two order reduces the result of product $\underline{Y}_{p h} \cdot \Delta \underline{\varphi}$ в (15), the value of error $\delta \underline{Y}_{p h}^{\text {nondis }}$ can be considered as tending to zero. For example, at $|\Delta \underline{\varphi}|$ equal to $10 \%$ of the direct sequence phase value module $\left(\left|\underline{U}_{A 1}^{\approx}\right|\right)$ and $\left|\underline{U}_{A 2}^{\approx}\right|$ equal to 4 $\%$ of $\left|\left(U_{A 1}^{\approx}\right)^{2}\right|$ the error of determination $\underline{Y}_{p h}^{\text {nondis }}$ of balanced load will have order of $-0,004 \cdot \underline{Y}_{p h}$ which corresponds in relative units to $-0.4 \%$.

Error $\delta \underline{Y}_{p h}^{d i s}$ taking into account (14) will be approximately determined as follows: 


$$
\delta \underline{Y}_{p h}^{d i s} \approx \underline{Y}_{p h} \cdot \Delta \underline{\varphi} \cdot\left(\frac{1}{\underline{U}_{p h}^{\approx}}+\frac{\underline{U}_{A 2}^{\approx}}{\left(\underline{U}_{A 1}^{\approx}\right)^{2}}\right) .
$$

Taking into account that $\underline{U}_{A 2}^{\approx} /\left(U_{A 1}^{\approx}\right)^{2}$ is the value of the second order with respect to $1 / \underline{U}_{p h}$, we can write:

$$
\delta \underline{Y}_{p h}^{d i s} \approx \underline{Y} p h \cdot \frac{\Delta \underline{\varphi}}{\underline{U_{p h}}} .
$$

From (17) it can be seen that the value of error $\delta \underline{Y}_{p h}^{d i s}$ will be determined by the value $\Delta \underline{\varphi}$. For example, at $|\Delta \underline{\varphi}|=0.1 \mid \frac{U_{p h}^{\approx}}{|c|}$ the order of the error $\delta \underline{Y}_{p h}^{d i s}$ is $0.1 \underline{Y}_{p h}$ at the true value $\underline{Y}_{p h}^{d i s}=0$. have:

At the unbalanced load with asymmetric voltage we

$$
\begin{aligned}
& \delta \underline{Y}_{p h}^{\text {nondis }}=\frac{1}{3} \cdot\left(\begin{array}{l}
\left.\underline{Y}_{A} \cdot\left|\frac{\Delta \underline{\varphi}}{\underline{U}_{A}^{\approx}}\right| \cdot e^{j\left(\alpha-\varphi_{A}\right)}+\underline{Y}_{B} \cdot\left|\frac{\Delta \underline{\underline{U}}}{\underline{U}_{B}}\right| \times\right) \\
\times e^{j\left(\alpha-\varphi_{B}\right)}+\underline{Y}_{C} \cdot\left|\frac{\Delta \underline{\varphi}}{\underline{U}_{C}^{\approx}}\right| \cdot e^{j\left(\alpha-\varphi_{C}\right)}
\end{array}\right) . \\
& \delta \underline{Y}_{p h}^{d i s}=\underline{Y}_{p h} \cdot \Delta \underline{\varphi} / \underline{U}_{p h}^{\approx}-\frac{1}{3} \cdot\left(\underline{Y}_{A}\left|\frac{\Delta \underline{\varphi}}{\underline{U}_{A}^{\approx}}\right| \cdot e^{j\left(\alpha-\varphi_{A}\right)}+\right. \\
& \left.+\underline{Y}_{B}\left|\frac{\Delta \underline{\underline{\varphi}}}{\underline{U}_{B}}\right| \cdot e^{j\left(\alpha-\varphi_{B}\right)}+\underline{Y}_{C}\left|\frac{\Delta \underline{\varphi}}{\underline{U}_{C}}\right| \cdot e^{j\left(\alpha-\varphi_{C}\right)}\right),
\end{aligned}
$$

where $\alpha=\arg (\Delta \underline{\varphi}) ; \varphi_{p h}=\arg \left(\underline{U}_{p h}^{\approx}\right)$.

Let us assume that phase voltages values $\left(\underline{U}_{A}^{\approx}, \underline{U}_{B}^{\approx}\right.$ и $\underline{U_{C}} \underset{\text { }}{ }$ ) are approximately symmetrical. Then, with the possibility of an arbitrary rotation of the system of vectors, we have:

$$
\begin{gathered}
\underline{Y}_{A}\left|\frac{\Delta \underline{\varphi}}{\underline{U}_{A}^{\approx}}\right| \cdot e^{j 0}+\underline{Y}_{B}\left|\frac{\Delta \underline{\varphi}}{\underline{U}_{B}^{\approx}}\right| \cdot e^{j 120}+\underline{Y}_{C}\left|\frac{\Delta \underline{\varphi}}{\underline{U}_{C}^{\approx}}\right| \cdot e^{j 240} \approx \\
\\
\approx \frac{|\Delta \underline{\varphi}|}{\left|\underline{\underline{U}_{p h}}\right|} \cdot\left[\underline{Y}_{A}^{d i s} \cdot e^{j 0}+\underline{Y}_{B}^{d i s} \cdot e^{j 120}+\underline{Y}_{C}^{d i s} \cdot e^{j 240}\right]
\end{gathered}
$$

Assuming that arguments of disturbing parts of conductivities $\left(\underline{Y}_{A}^{d i s}, \underline{Y}_{B}^{d i s}\right.$ and $\left.\underline{Y}_{C}^{d i s}\right)$ are such that their multiplication on unit vectors $e^{j 0}, e^{j 120}$ and $e^{j 240}$ in a sum give a vector $\left|\underline{Y}_{A}^{d i s}\right|+\left|\underline{Y}_{B}^{d i s}\right|+\left|\underline{Y}_{B}^{d i s}\right|$, the error module $\delta \underline{Y}_{p h}^{\text {nondis }}$ for the case of unbalanced load can be estimated by the following inequality:

$$
\left|\delta \underline{Y}_{p h}^{\text {nondis }}\right|<\frac{|\Delta \underline{\varphi}|}{\left|\underline{U_{p h}^{\approx}}\right|} \cdot\left|\underline{Y}_{p h \max }^{\text {dis }}\right|,
$$

where $\underline{Y}_{p h \max }^{d i s}$ is the maximal phase disturbing conductivity which for the limit case (asymmetric load with star connection (Fig.3) set by one shoulder)equals to:

$$
\underline{Y}_{p h \max }^{\text {dis }}=\frac{2}{3} \underline{Y}_{p h} \text {. }
$$

So, for $|\Delta \underline{\varphi}|=0.1 \quad\left|\underline{U}_{p h}^{\approx}\right|$ we have $\left|\delta \underline{Y}_{p h}^{\text {nondis }}\right|<0,067 \cdot\left|\underline{Y}_{p h}\right| \cdot$

Let us consider the error $\delta \underline{Y}_{p h}^{d i s}$ taking into account that the second summand in (19) is $\delta \underline{Y}_{p h}^{\text {nondis }}$ (21):

$$
\delta \underline{Y}_{p h}^{d i s} \approx \frac{\Delta \underline{\varphi}}{\underline{U}_{p h}^{\approx}} \cdot\left(\underline{Y}_{p h}-\delta \underline{Y}_{p h}^{\text {nondis }}\right) .
$$

Taking into account (22), the module of the maximum error value $\delta \underline{Y}_{p h}^{d i s}$ can be estimated by the following inequality:

$$
\left|\delta \underline{Y}_{p h \max }^{\text {dis }}\right|<\frac{|\Delta \underline{\varphi}|}{\left|\underline{U}_{p h}^{\approx}\right|} \cdot \frac{5}{3}|\underline{Y} p h| .
$$

Quantitatively, at $|\Delta \underline{\varphi}|=0.1 \quad\left|\underline{U_{p h}}\right|$, $\left|\delta \underline{Y}_{p h \max }^{\text {dis }}\right|<0,17 \cdot|\underline{Y} p h|$.

From the carried out analysis of errors $\delta \underline{Y}_{p h}^{\text {nondis }}$ and $\delta \underline{Y}_{p h}^{d i s}$ it follows that disturbing parts of conductivities of balanced and unbalanced generalized load can be determined with high accuracy. Determination of disturbing parts is characterized by significant errors commensurate with the exact values of the unknown quantities.

\section{Conclusions.}

The structure of the equivalent circuit of substitution generalized linear loads in three-phase three-wire network is determined by three independent units, which corresponds to the connection diagram of elements in a triangle. The exact definition of the equivalent circuit parameters based on the methodology for measuring the quality parameters of electric energy is not technically feasible. A method for the approximate determination of the parameters of the equivalent circuit of substitution generalized linear load based on an approximate determination of the phase voltages. Errors calculating nondisturbing parts equivalent circuit parameters of generalized linear substitution loads are characterized by small quantities, which can be neglected. Errors calculation distorting parts may reach values comparable with the exact values.

\section{REFERENCES}

1. Kartashev I., Tulsky V., Shamonov R. Assessing of impact of disturbing load on power quality. 17th International Conference on Electricity Distribution. Barcelona, 12-15 May, 2003. pp. 1-5.

2. Zhezhelenko I.V., Shidlovskiy A.K., Pivnyak G.G., Sayenko Yu.L., Noyberger N.A. Elektromagnitnaya sovmestimost potrebiteley [Electromagnetic compatibility of customers]. Moscow, Mashinostroenie Publ., 2012. 349 p. (Rus). 
3. Chepmen D. Price of low power quality. Energosberezhenie - Energy Saving, 2004, no.1, pp. 66-69. (Rus).

4. Sayenko Yu., Kalyuzhniy D. Analytical methods for determination of the factual contributions impact of the objects connected to power system on the distortion of symmetry and sinusoidal waveform of voltages. Przeglad Elektrotechniczny, 2015, vol.11, pp. 81-85. doi: 10.15199/48.2015.11.23.

5. Gamazin S.I., Petrovich V.A. Determining the actual contribution of the consumer in the distortion of the quality parameters of electric energy. Promyishlennaya energetika, 2003, no.1, pp. 38-42. (Rus).

6. Aiqiang Pan, Jian Zhou. Power quality analysis and harmonic tracing in city grid based on big monitoring data. $23 \mathrm{rd}$ International Conference on Electricity Distribution. Lyon, 1518 June 2015. pp. 1-4.

7. Sayenko Y., Sukhonos M., Kalyuzhniy D., Bolgov V. Mathematical model for real-time assessment of contributions of disturbing sources to power quality level at a Point of Common
Coupling. 10th Electric Power Quality and Supply Reliability (PQ) Conference, Tallinn, 29-31 August, 2016, pp. 29-35. doi: 10.1109/pq.2016.7724085.

Received 29.09.2016

Yu.L. Sayenko ${ }^{1}$, Doctor of Technical Science, Professor,

D.N. Kalyuzhniy ${ }^{2}$, Candidate of Technical Science, Associate Professor,

${ }^{1}$ Pryazovskyi State Technical University,

7, Universytets'ka Str., Mariupol, 87500, Ukraine,

phone+380629 446551, e-mail: YuriSayenko@mail.ru

${ }^{2}$ O.M. Beketov National University of Urban Economy in Kharkiv,

12, Revolution Str., Kharkiv, 61002, Ukraine,

phone+38050 5606835, e-mail: KalyuzhniyDN@mail.ru

\section{How to cite this article:}

Sayenko Yu.L., Kalyuzhniy D.N. Identification of generalized linear load in three-phase three-wire network in the problem of the distribution of actual contributions at the point of common coupling. Electrical engineering \& electromechanics, 2016, no.6, pp. 65-69. doi: 10.20998/2074-272X.2016.6.11. 